\title{
(Fortsetzung des Inhaltsverzeichnisses)
}

Petit, P.: Libanius et la vie municipale à Antioche au IVe siècle après J.-C. (O. Ei Bf eld t) . . . 428

Ritter, H.: Karagös, hrsg. u. erklärt. Mit Beitr. v. A. Tietze (J. Németh) . . . . . . . . 466

Schäfer, E.: Unter Räubern in Tibet (J. Schubert) 489

Schaeffer, C. F.-A., et Ch. Virolleaud: Reprise des Fouilles de Ras Shamra-Ugarit. Campagnes XII à XVII (O. EiBfeldt). • . . . . . 445

Schramm, G.: Drogen und Heilpflanzen der altchinesischen Materia medica in der Therapie der Lungentuberkulose (F. H übot ter) . . . . 488

- Uber die chinesische Moxibustion (F. Hübotter) 488

- u. E. Seidel: Tierheilkunde im alten China (F. Hübotter) . . . . . . . . . 488

Schurmann, H. F.: Economic Structure of the Yüan Dynasty (P. Ratchnevsky) . . . . . 481

Seidel, E., 8. Schramm, G.

Selms, A.van: De Rol der Lofprijzingen (O. Ei Bfeldt) ............. 454

Tietze, A., s. Ritter, H.

Vanoverbergh, M.: Songs in Lepanto-Igorot as it is spoken at Bauko (H. Kähler) . . . . . . 480

Virolleaud, Ch., s. Schaeffer, C. F.-A.

Wackernagel, J.: Altindische Grammatik. II, 1 (M. Mayrhofer) . . . . . . . . 474
Wångstedt, St. V.: Ausgewählte demotische Ostraka (F. Lexa) . . . . . . . . . . . . . . . . . . . . 439 Yadin, Y., s. Avigad, N.

\section{Systematisehe Ubersicht:}

Aufsätze . . . . . . . . . . . . 421-426

Allgemeines . . . . . . . . . 426-432

Ägyptologie . . . . . . . . . . . . . 432-444

Keilschriftforschung . . . . . 445-450

Altes Testament, Neues Testament, Spătjudentum, Urchristentum .... . 450-456 Semitistik, Islamistik . . . . . 456-464

Kaukasus, Turkologie, Iran . . . . . . 464-469 Südasien. . . . . . . . . . 469-481

Zentral- und Ostasien $481-495$

Afrikanistik ... 495-496

Zeitschriftenschau: Journal of Near Eastern Studies - Kirjath Sepher - Language Revue Biblique - Revue de l'Histoire des Religions - Rocznik Orientalistyczny Semitica - Studi e materiali di storia delle religioni - Studies in Bibliography and Booklore - Sumer, A Journal of Archaeology in Iraq - Tsing Hua Journal of Chinese Studies . . . . . . . 496-508

\section{Neuerscheinungen :}

Albright, W. F.: Die Bibel im Licht der Altertumsforschung. Ein Bericht über die Arbeit eines Jahrhunderts. Mit einer Zeittafel. Vorwort von Prof. D. EiBfeldt. Stuttgart: Calwer Verlag [1957]. 148 S. $8^{\circ}$. Kart. DM 6.80; Lw. 8.80.

Al-Harawi: Guide des Lieux de Pèlerinage. Trad. J. Sourdèl-Thomine. Damas: Institut Français de Damas 1957. LV, 231 S. $8^{0}$.

L'Ancien Testament et l'Orient. Études présentées aux VIes Journées Bibliques de Louvain (11.-13. sept. 1954). Louvain: Université de Louvain, Inst. Orientaliste 1957. IV, $231 \mathrm{~S} .4^{\circ}=$ Orientalia et Biblica Lovaniensia I.

Berque, J.: Al-Yousi. Problèmes de la Culture Marocaine au XVIIeme siècle. Paris: Mouton 1958. 144 S., 4 Taf. gr. $8^{\circ}=$ École Pratique des Hautes Études, SectionVI: Le Monde d'Outre-Mer passé et présent, Serie I: Études II.

Bittel, K., Naumann, R., Beran, T., Hachmann, R., u. G. Kurth: Boğazköy III. Funde aus den Grabungen 1952-1955. Berlin: Gebr. Mann 1957. 71 S. m. 16 Abb., 14 Falttaf., 24 Kunstdrucktaf. $2^{\circ}=$ Ausgrabungen der Deutschen Orient-Gesellschaft und des Deutschen Archäologischen Instituts. Abhandl. der Deutschen Orient-Gesellschaft Nr. 2. Kart. DM 30.-.

Cowan, D.: An Introduction to Modern Literary Arabic. London: Cambridge University Press 1958. XI, $205 \mathrm{~S}$. $8^{\circ} .35 \mathrm{~s}$.

Davies, N. de Garis $\dagger$ : A Corpus of inscribed Egyptian Funerary Cones. Ed. by H. M. Laming MacAdam. Part. I: Plates. Oxford: Griffith Inst./University Press 1957. XX S., $611 \mathrm{Abb}$. auf Taf. $2^{\circ}$.

Davies, Nina M.: Picture Writing in Ancient Egypt. London: Oxford University Press 1958, $56 \mathrm{~S}$., $17 \mathrm{Taf} .4^{\circ}, 30 \mathrm{~s}$

Duchesne-Guillemin, J.: The Western Response to Zoroaster. Oxford: Clarendon Press; London: Oxford University Press 1958. VII, 112 S. $8^{\circ}=$ Ratanbai Katrak Lectures 1956. $25 \mathrm{~s}$.

Eberhard, O.: Aus Palästinas Legendenschatz. Uberlieferungen und Erläuterungen aus der jüdischen und der arabisch-islamischen Welt, bearb. u. hrsg. Berlin: Evang. Verlagsanstalt [1958]. 303 S. $8^{\circ}$. DM 10.80.

Eliade, M.: Schamanismus und archaische Ekstasetechnik. Zürich/Stuttgart: Rascher 1957. VIII, $472 \mathrm{~S}$. gr. $8^{\circ}$. Lw. DM 29.50.
Friedrich, J.: Hethitisches Wörterbuch. Kurzgefaßte kritische Sammlung der Deutungen hethitischer Wörter. 1. Ergänzungsheft. Heidelberg: Winter [1957]. V, $42 \mathrm{~S}$. gr. $8^{\circ}=$ Indogermanische Bibliothek, 2. Reihe: Wörterbücher. DM 8.-.

Gabain, A. von, u. W. Winter: Türkische Turfantexte IX. Ein Hymnus an den Vater Mani auf ,Tocharisch" B mit alttürkischer Ubersetzung. Berlin: Akademie-Verlag 1958. $45 \mathrm{~S} ., 2 \mathrm{Taf} .4^{\circ}=$ Abhandl. der Deutschen Akademie der Wissenschaften zu Berlin, Klasse für Sprachen, Literatur und Kunst, Jahrg. 1956, Nr. 2. DM 12.50.

[Gaster, M.:] Gaster Centenary Publication, ed. by B. Schindler. London: Lund, Humphries \& Co. 1958. VI, 40 S., 1 Porträt gr. $8^{\circ} .10 \mathrm{s.} 6 \mathrm{~d}$.

Heissig, W., u. C. R. Bawden: Mongrol Borjigid Obor-Un Teüke von Lomi (1732). - Meng-Ku Shih-Hsi-P'u. Hrsg. u. mit Einleitungen versehen. Wiesbaden: Harrassowitz 1957. VI, $133 \mathrm{~S}$. gr. $8^{\circ}=$ Göttinger Asiatische Forschungen Bd. 9. DM 14.--

Herrmann, F., u. P. Germann: Beiträge zur afrikanischen Kunst. Die afrikanische Negerplastik als Forschungsgegenstand. Von F. Herrmann. Negerplastiken aus dem Museum für Völkerkunde zu Leipzig. Von P. Germann. Berlin: Akademie-Verlag 1958. VII, $59 \mathrm{~S}$., $1 \mathrm{Kte}, 36 \mathrm{Taf}$. gr. $\mathbf{8}^{0}=$ Veröffentl. des Museums für Völkerkunde zu Leipzig, H 9. DM 8.-.

Indien. Entwicklung seiner Wirtschaft und Kultur. Unter Leitung von Prof. Dr. Edgar Lehmann bearb. von Dr. Hildegard Weisse. Leipzig: Verlag Enzyklopädie 1958. III, $18 \mathrm{~S}$. Text, 90 farb. Ktn. auf 16 Taf. $2^{0}=$ Historisch-geographisches Kartenwerk I. DM 55.-. Jansma, T.: Investigations into the early Syrian Fathers on Genesis. Leiden: Brill 1958. S. 69-181. gr. $8^{\circ}=$ Oudtestamentische Studiēn Deel XII.

Kamil, M.: Amharische Kaiserlieder. Aufgezeichnet, umschrieben und übersetzt. Wiesbaden: F. Steiner in Komm. 1957. VI, 50 S. gr. $8^{0}=$ Abhandl. für die Kunde des Morgenlandes, hrsg. v. d. Deutschen Morgenländischen Gesellschaft XXXII, 4. DM 10.-.

Kluge, Inge-Lore: Miyoshi Kiyoyuki. Sein Leben und seine Zeit. Berlin: Akademie-Verlag 1958. $80 \mathrm{~S} .4^{0}=$ Deutsche Akademie der Wissenschaften zu Berlin, Inst. 1. Orientforsch., Veröff. Nr. 35. DM 29.-.

Forts. s. vorletzte Seite 